\title{
APLICABILIDADE DO IMC DE TREFETHEN EM ESCOLARES
}

Miguel Altamir Ribas Junior*, Luis Paulo Gomes Mascarenhas**, Michele Cordova*, Valderi Abreu de Lima***, Marcos Tadeu Grzelczak $^{* * *}$, William Cordeiro de Souza****

Autor correspondente: William Cordeiro de Souza - williammixx@hotmail.com

* Graduando em Educação Física pela Universidade do Contestado

** Doutor em Saúde da Criança e do Adolescente. Professor da Universidade Estadual do Centro-Oeste

*** Mestrando em Educação Física pela Universidade Federal do Paraná

**** Núcleo de Estudos em Atividade Física - NEAF. Universidade do Contestado - UnC

\section{Resumo}

Introdução: Vários métodos antropométricos vêm sendo desenvolvidos com o intuito de avaliar o estado nutricional tanto de adultos, quanto de crianças e adolescentes. Objetivo: Verificar o nível de aplicabilidade do IMC proposto por Trefethen, comparado a outras técnicas indiretas de avaliação da composição corporal. Métodos: A amostra foi constituída por 58 escolares $(28$ meninos e 30 meninas) de 10 a 14 anos de idade, do município de São Bento do Sul-SC. Para avaliação do estado nutricional foi avaliado a massa corporal e a estatura. Através desses dados foi calculado o IMC utilizado às formulas: IMC Quetelet= Massa Corporal/Estatura ${ }^{2}$ e IMC Trefethen= Massa corporal $(\mathrm{Kg})$ $\mathrm{x} 1,3$ /altura $(\mathrm{m})^{2,5}$. Coletaram-se as dobras cutâneas do tríceps e subescapular para avaliar o \%G, para isso utilizado o protocolo de Lohman (1986). A normalidade dos dados foi realizada pelo teste de Kolmogorov Smirnov. Após, realizou-se a estatística descritiva e o fator de correlação de Pearson ( $r$ ) foi realizado para verificar as associações entre o IMC (Trefethen), IMC (Quetelet) e o \%G. Foi adotado um nível de significância de $p<0,05$. Resultados: Foi encontrada uma relação muito forte e significante entre o IMC de Trefethen e o IMC de Quetelet $(r=0,97 ; p=<0,001)$. Já entre o IMC de Trefethen e o\%C foi verificada uma relação forte e significante $(r=0,72 ; p=<0,001)$. Conclusão: Diante disso, conclui-se que a nova equação do IMC pode ser considerada como um novo método para avaliar o estado nutricional de crianças e adolescentes.

Palavras-chave: Aplicabilidade; Estado Nutricional; Escolares. 


\title{
BMI APPLICABILITY OF TREFETHEN IN SCHOOL
}

\begin{abstract}
Introduction: Several anthropometric methods have been developed in order to evaluate the nutritional status of both adults as children and adolescents. Objective: To determine the applicability level of BMI proposed by Trefethen, compared to other indirect methods of assessing body composition. Methods: The sample consisted of 58 students ( 28 boys and 30 girls) from 10 to 14 years old, the city of São Bento do Sul-SC. To assess the nutritional status was assessed by body mass and height. Through these data we calculated the BMI used the formulas: BMI Quetelet= body mass/height ${ }^{2}$ and BMI Trefethen= body weight ( $\left.\mathrm{kg}\right) \times 1.3 /$ height $(m)^{2.5}$. They were collected skinfolds triceps and subscapularis to evaluate the \%BF, for it used Lohman Protocol (1986). The normality of the data was performed using the Kolmogorov-Smirnov test. After there was the descriptive statistics and Pearson correlation factor ( $r$ ) was performed to verify the associations between BMI (Trefethen), BMI (Quetelet) and \%BF. Ap significance level of $<0.05$ was adopted. Results: A very strong and significant relationship between Trefethen BMI and BMI Quetelet was found $(r=0.97, p=<0.001)$. Among the Trefethen BMI and\% BF was found a strong and significant correlation $(r=0.72, p=<0.001)$. Conclusion: Therefore, it is concluded that the new equation of BMI can be considered as a new method for assessing the nutritional status of children and adolescents.
\end{abstract}

Keywords: Applicability; Nutritional Status; School.

\section{INTRODUÇÃO}

O excesso de gordura corporal é considerado um dos maiores problemas de saúde pública em muitos países, especialmente os mais industrializados. Nas últimas duas décadas este quadro de crescente obesidade populacional também passou a preocupar países em desenvolvimento, como por exemplo, o Brasil, dentre elas, destaca- se a população escolar.(1)

Os altos índices de obesidade têm alcançado números elevados e isso acaba contribuindo para o risco de desenvolvimento precoce de doenças crônicas degenerativas, tanto em crianças e adolescentes, bem como em adultos. ${ }^{(2)}$

Conforme destaca Souza et al. ${ }^{(3)}$ a progressão da transição nutricional é caracterizada pela ocorrência mais expressiva de sobrepeso e obesidade, que está aumentado consideravelmente, uma vez que a população, especialmente a escolar está mudando seus hábitos alimentares, e essas mudanças manifesta-se diretamente no estado nutricional.

Para Queiroga,(4) a obesidade é caracterizada pela presença de uma maior quantidade de gordura corporal, e ressalta que para avaliar o sobrepeso/obesidade deve ser empregados métodos com cautela, pois muitos deles, como por exemplo, o índice de massa corporal (IMC) ele não leva em consideração a composição do corpo ou, mais exatamente a quantidade de gordura presente.

Apesar do IMC (Quetelet) sofrer várias críticas, esse método é muito utilizado em estudos epidemiológicos, pois é uns dos métodos mais baratos, tem fácil aplicabilidade, e menos constrangimentos para os avaliados, e contribui para uma mensuração mais rápida comparando com os demais métodos de avaliação da composição corporal.(5) 
A grande preocupação em relação à utilização do IMC é para identificar a obesidade e/ ou excesso de peso estaria no fato de que a quantidade de gordura poderia demonstrar baixa relação com o peso. No entanto, em estudos epidemiológicos, devido à grande amplitude dos índices de obesidade é reduzido. Por outro lado, esse erro aumenta quando se deseja analisar um único indivíduo ou pequenos grupos. ${ }^{(4)}$

O IMC limita-se em não diferir a massa magra da massa gorda, e isso pode ser observado em um atleta com grande quantidade de massa magra o que resulta em um IMC acima do desejável, isso por conta da massa magra e não do excesso de gordura. ${ }^{(6)}$

Devido ao IMC apresentar baixa sensibilidade em detectar indivíduos com excesso de gordura corporal, no ano de 2013 na Inglaterra, o professor Floyd Nicholas Trefethen, propôs uma nova equação para o IMC, a qual apresenta uma melhor aproximação do tamanho e das formas corporais. Destacando que a nova da formula representa as dimensões corporais de maneira errônea, uma vez que pessoas de maior estatura possuem compartimentos estruturais e fisiológicos maiores que pessoas de menor estatura. ${ }^{(7,8)}$

Sendo assim, o presente estudo buscou como objetivo verificar o nível de aplicabilidade do IMC proposto por Trefethen, comparado a outras técnicas indiretas de avaliação da composição corporal (IMC - Quetelet e \%Gordura (\%G)) em escolares de 10 a 14 anos de idade.

\section{MÉTODOS}

A amostra intencional foi constituída por 58 escolares ( 28 meninos e 30 meninas) entre 10 e 14 anos de idade, matriculados em uma escola do ensino fundamental, do município de São Bento do Sul-SC.

Foram excluídos do estudo adolescentes portadores de doenças crônicas que interferissem diretamente com o peso e a estatura e aqueles que, no momento da avaliação, tivessem algum impedimento para a obtenção das medidas antropométricas, que não compareceram na escola nos dias marcados para coleta de dados, e os adolescentes cujos pais não autorizaram a participação. Sendo também respeitada a vontade dos adolescentes que se recusaram a participar mesmo com a autorização dos pais.

Os escolares somente foram avaliados mediante assinatura do Termo de Consentimento Livre e Esclarecido pelos pais e responsáveis, conforme as normas éticas exigidas pela Resolução $n^{\circ}$ 466/2012 do Conselho Nacional de Saúde. Este trabalho foi aprovado pelo Comitê de Ética da Universidade do Contestado - UnC (número do Parecer: CAAE 05020512.7.0000.0117).

A avaliação antropométrica consistiu na mensuração da massa corporal e estatura. $\mathrm{Na}$ avaliação da massa corporal o avaliado deveria se posicionar em pé, de costas para escala da balança, usando o mínimo de roupa. ${ }^{(9)} \mathrm{Na}$ avaliação da massa corporal foi utilizada uma balança digital da marca Techline, (fabricada no Brasil) devidamente calibrada, com graduação de 100 gramas e escalas variando de 0 a $180 \mathrm{Kg}$.

A estatura foi identificada pelo maior valor entre o vértex e a região plantar obedecendo ao plano de Frankfurt. ${ }^{\left({ }^{9}\right)}$ A medida foi verificada através de uma trena flexível marca Sanny Medical Sparrett, (fabricada no Brasil), fixada na parede lisa, com 3 metros e graduação de $0,1 \mathrm{~cm}$ com o zero coincidindo com o solo.

Através destes dados foi calculado o IMC, proposto por Trefethen ${ }^{(8)}$ e o IMC sugerido por Quételet ${ }^{(10)}$ utilizado às seguintes formulas: IMC Trefethen= Massa corporal $(\mathrm{Kg}) \times 1,3$ /altura $(\mathrm{m})^{2,5}$ e IMC Quetelet $=$ Massa Corporal/Estatura ${ }^{2}$.

Para a classificação do IMC utilizou-se como referência as curvas de percentis, recomendado pelo Center for Disease Control and Prevention, ${ }^{(11)}$ que classifica como baixo IMC para idade valores $<$ percentil 5, IMC adequado ou eutrófico $>$ percentil 5 e $<$ percentil 85 , sobrepeso $>$ percentil 85 e $<$ percentil 95 e para obesidade valores $>$ percentil 95 . 
Através desses dados foi classificado o sobrepeso e obesidade nas crianças.

Para avaliar o \%G, foi utilizado o protocolo de Lohman ${ }^{(12)}$ que sugere a soma das Dobras Cutâneas (DC) do Tríceps e Subescapular (TR $\pm S E$ ), convertendo esse valor em \%GC através da seguinte constante para meninos e meninas $(<35 \mathrm{~mm})$ : $\% \mathrm{G}=1,35(\mathrm{TR} \pm \mathrm{SE})-0,012(\mathrm{TR} \pm \mathrm{SE})^{2}-\mathrm{C}$.

Inicialmente, realizou-se a coleta da dobra cutânea tricipital, onde a referência anatômica foi o processo acromial da escápula e o processo olecrano da ulna. Com o indivíduo em pé e braços relaxados ao longo do corpo foi medida a dobra na face posterior do braço, na distância média entre a borda súperolateral do acrômio e o bordo inferior do olecrano. Sua determinação foi realizada seguindo o eixo longitudinal do membro. ${ }^{(13)}$

$\mathrm{Na}$ determinação da dobra cutânea de subescapular o avaliado deveria permanecer em pé e ereto com os braços ao longo do corpo. A dobra cutânea foi pinçada obliquamente a partir do ponto de re- ferência escapular, num ângulo determinado pela dobra natural da pele. ${ }^{(13)}$ Para a classificação do \%G foi utilizado como referência os pontos de corte sugeridos por Lohman. ${ }^{(14)}$

Para verificar a normalidade dos dados foi utilizado o teste de Kolmogorov Smirnov. Visto que a distribuição foi normal, realizou-se a estatística descritiva (média, desvio padrão e a (\%) frequência percentual). O fator de correlação de Pearson ( $r)$ foi realizado para verificar as associações entre o IMC (Trefethen), IMC (Quetelet) e o \%G. Foi adotado um nível de significância de $p<0,05$. As análises foram realizadas por meio do pacote estatístico BioEstat 5.0.

\section{RESULTADOS}

A tabela 1 apresenta os dados de idade, massa corporal, estatura, IMC (Trefethen), IMC (Quetelet), DC tricipital, DC subescapular, \%G para caracterização da amostra.

Tabela 1 - Caracterização da amostra

\begin{tabular}{|c|c|c|}
\hline VARIÁVEIS & MÉDIA & DEsVIo PADRÃo \\
\hline Idade (anos) & 11,8 & $\pm 1,41$ \\
\hline Massa Corporal (kg) & 47,32 & $\pm 10,12$ \\
\hline Estatura (m) & 1,50 & $\pm 0,09$ \\
\hline IMC $\left(\mathrm{Kg} \times 1,3 /\right.$ altura $(\mathrm{m})^{2,5}$ & 21,98 & $\pm 3,37$ \\
\hline IMC $\left(\mathrm{kg} / \mathrm{m}^{2}\right)$ & 20,72 & $\pm 3,25$ \\
\hline DC Tríceps (mm) & 12,55 & $\pm 5,41$ \\
\hline DC Subescapular (mm) & 10,86 & $\pm 6,03$ \\
\hline$\% G$ & 22,53 & $\pm 8,54$ \\
\hline
\end{tabular}

O gráfico 1 apresenta a relação entre o IMC de Trefethen e o IMC de Quetelet, e se pode perceber uma relação muito forte e significante entre as variáveis analisadas $(r=0,97 ; p=<0,001)$. 


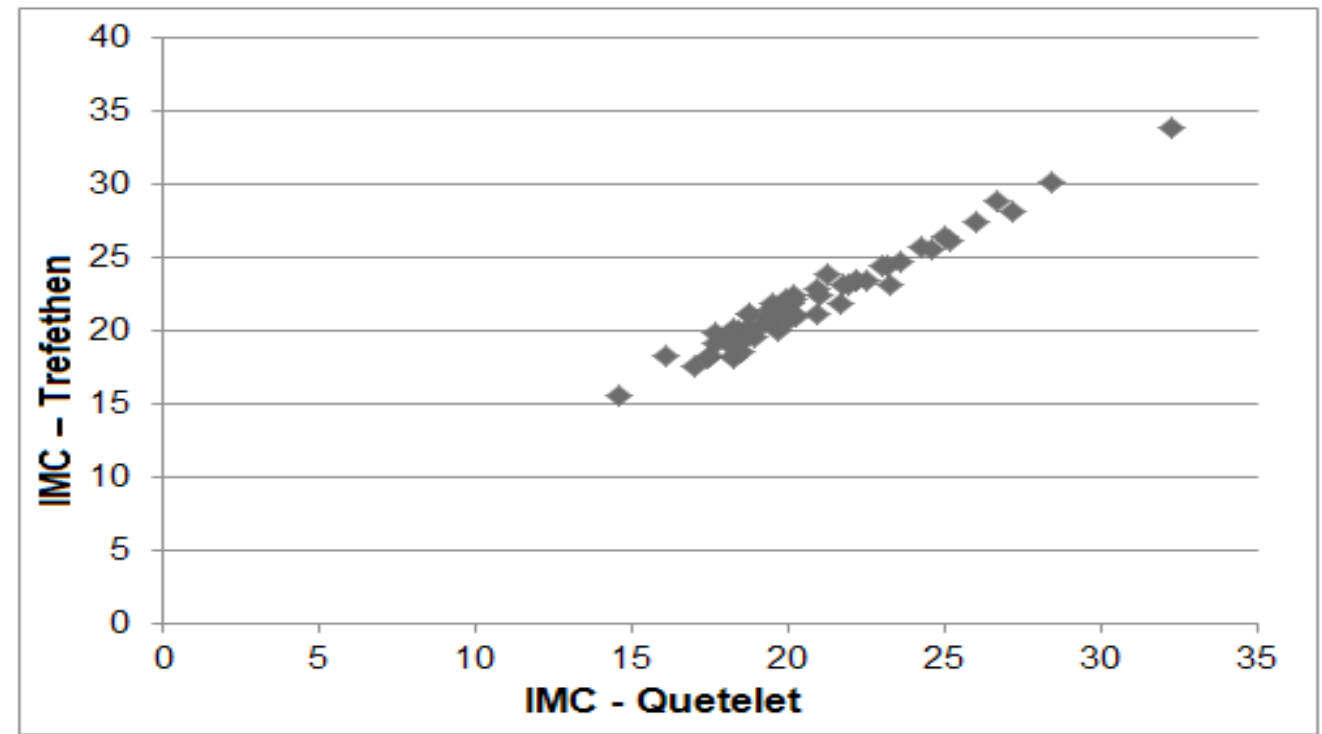

Gráfico 1 - Relação entre o IMC de Trefethen e o IMC de Quetelet

Já o gráfico 2 apresenta a relação entre o IMC de Trefethen e o \%G, e se pode perceber uma relação forte e significante entre as variáveis analisadas $(r=$ $0,72 ; p=<0,001)$.

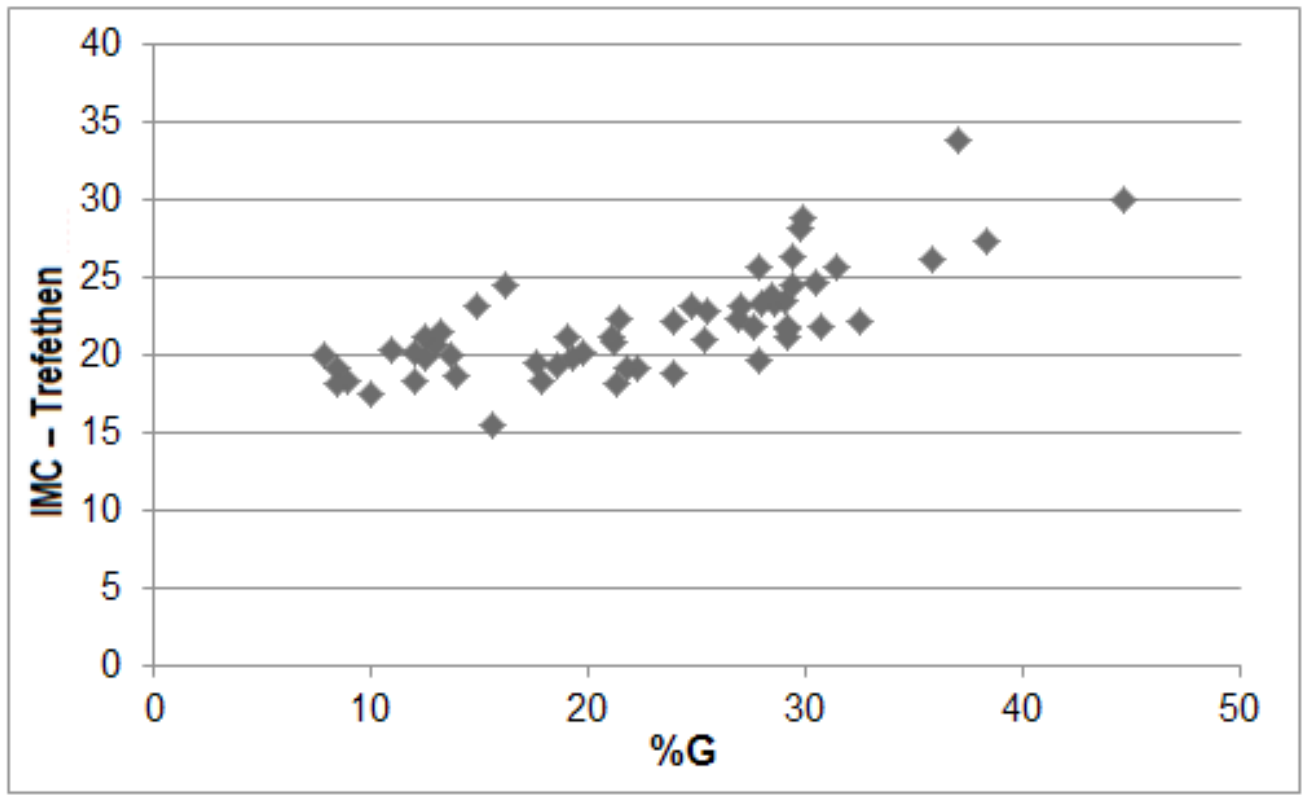

Gráfico 2 - Relação entre o IMC de Trefethen e o \%G

A tabela 2 apresenta os dados obtidos nas clas- sificações do IMC de Trefethen, IMC de Quetelet e o\%G corporal. 
Tabela 2 - Classificações do IMC de Trefethen, IMC de Quetelet e do \%G

\begin{tabular}{|c|c|c|c|}
\hline ClassificaÇÃo & MENINOS & MENINOS & TOTAL \\
\hline \multicolumn{4}{|l|}{ IMC - Trefethen } \\
\hline Eutrófico & $n=22(78,57 \%)$ & $n=25(83,33 \%)$ & $n=47(81,04 \%)$ \\
\hline Sobrepeso & $\mathrm{n}=4(14,28 \%)$ & $n=4(13,33 \%)$ & $n=8(13,79 \%)$ \\
\hline Obesidade & $\mathrm{n}=2(7,15 \%)$ & $n=1(3,34 \%)$ & $n=3(5,12 \%)$ \\
\hline \multicolumn{4}{|l|}{ IMC - Quetelet } \\
\hline Eutrófico & $n=25(89,28 \%)$ & $n=25(83,33 \%)$ & $n=50(86,20 \%)$ \\
\hline Sobrepeso & $n=3(10,72 \%)$ & $n=4(13,33 \%)$ & $\mathrm{n}=7(12, \mathrm{O} 6 \%)$ \\
\hline Obesidade & $\mathrm{n}=\mathrm{O}(\mathrm{O}, \mathrm{O} \% \%)$ & $n=1(3,34 \%)$ & $n=1(1,72 \%)$ \\
\hline \multicolumn{4}{|l|}{ \%G Corporal } \\
\hline Muito Baixo & $\mathrm{n}=\mathrm{O}(\mathrm{O}, \mathrm{O} \% \%)$ & $\mathrm{n}=\mathrm{O}(\mathrm{O}, \mathrm{O} \% \%)$ & $\mathrm{n}=\mathrm{O}(\mathrm{O}, \mathrm{O} \% \%)$ \\
\hline Baixo & $n=6(21,42 \%)$ & $n=O(O, O O \%)$ & $n=6(10,34 \%)$ \\
\hline Nível Ótimo & $n=13(46,42 \%)$ & $n=11(36,66 \%)$ & $n=24(41,37 \%)$ \\
\hline Moderadamente Alto & $\mathrm{n}=2(7,14 \%)$ & $n=11(36,66 \%)$ & $n=13(22,41 \%)$ \\
\hline Alto & $n=6(21,42 \%)$ & $n=5(16,66 \%)$ & $n=11(18,96 \%)$ \\
\hline Muito Alto & $n=1(3,57 \%)$ & $n=3(10,00 \%)$ & $n=6(6,89 \%)$ \\
\hline
\end{tabular}

\section{DISCUSSÃO}

O presente estudo com o intuito de verificar o nível de aplicabilidade do IMC proposto por Trefethen, comparado a outras técnicas indiretas de avaliação da composição corporal (IMC - Quetelet e \%Gordura (\%G)), em escolares de 10 a 14 anos de idade encontrou uma relação muito forte e significante entre IMC de Trefethen e o IMC de Quetelet ( $r=$ $0,97 ; p=<0,001)$.

Dados semelhantes foram encontrados no estudo de Westphal et al.(15) que tiveram por objetivo relacionar o IMC de Quetelet e o IMC Trefethen em acadêmicos de educação física, com idades entre 18 e 58 anos e encontraram uma relação muito forte e significante $(r=0,99 ; p=<0,001)$; com um intervalo de confiança de IC 95\%=0,96-0,99 entre os métodos.

O mesmo ocorreu em estudo de Beaudin e Ska$\mathrm{za}^{(16)}$ que tinham por intuito analisar os fatores influentes da obesidade e depressão em adultos, quando relacionaram o IMC novo (Trefethen) com - IMC velho (Quetelet) observaram uma relação muito forte $(r=0,98)$.

$\mathrm{Na}$ validação da equação apresentada por Trefethen ${ }^{(8)}$ foi realizada uma análise de regressão com a equação de Quetelet e foi constatada uma correlação positiva e perfeita $(r=1,0)$ entre às equações.

Segundo Westphal et al. ${ }^{(15)}$ essa relação muito forte e perfeita encontrada no referido estudo e nos mencionados acima, provavelmente foi provocada pelo motivo de relacionar duas variáveis idênticas, no caso o IMC. Essa afirmação pode ser observada na tabela 1 (caracterização da amostra) e na tabela 2 (classificação dos métodos) onde os valores expressos são semelhantes em ambos os métodos do IMC.

Quanto à relação entre o IMC de Trefethen e o \%G foi encontrada uma correlação forte e signifi- 
cante $(r=0,72 ; p=<0,001)$. Cabe mencionar que a literatura cientifica (base de dados), tanto nacional quanto a internacional limita-se em estudos que correlacionem à nova formula com o \%G corporal, assim inviabilizando a discussão do estudo.

A tabela 2 apresenta que grande maioria dos avaliados apresentou \%G corporal classificado como ótimo, tanto para o gênero masculino quanto para o feminino e no grupo geral, respectivamente. Seguido de moderadamente alto, alto, baixo e muito alto. Vale destacar que nenhum avaliado foi classificado com \%G corporal muito baixo.

A nova equação do IMC apresenta-se como uma nova ferramenta para avaliar o estado nutricional tanto de adultos, quanto de crianças, onde essa formula objetiva-se em obter dados mais próximos da realidade. ${ }^{(17,18)}$

Em relação à equação proposta por Quételet ${ }^{(10)}$ a mesma apresenta controversa quanto a sua sensibilidade e especificidade. Nunes ${ }^{(19)}$ argumenta que o IMC (Quetelet) é um dos métodos antropométricos mais adequados para estimar o sobrepeso e obesidade de crianças e adolescentes, pois o mesmo apresenta-se como um ótimo indicador de doenças cardiovasculares.

Lohman ${ }^{(20)}$ não recomenda abandonar o uso do IMC, muito menos adotá-lo como uma única medida em estudos epidemiológicos ou sobre o perfil da composição corporal, crescimento e desenvolvimento infantil.

Ambos os métodos de IMC (Quetelet e Trefethen) apresentaram valores médios semelhantes $\mathrm{e}$ de classificação, diante dos resultados apresentados pode-se destacar que os dois métodos apresentam boa aplicabilidade e sensibilidade para avaliar o sobrepeso e obesidade de escolares.

O presente estudo limitou-se em não caracterizar o nível de atividade física praticada pela amostra avaliada, bem como a classe socioeconômica, possivelmente estes fatores podem ter influenciado nos achados do estudo. Desta forma, sugere-se que novos estudos sejam realizados com distintas populações utilizando a nova equação, correlacionando-a com outros métodos antropométricos, para assim dar mais fidedignidade ao novo método de avaliação de estado nutricional.

\section{CONCLUSÃO}

Ao finalizar o estudo constatou-se que a nova formula do IMC apresentou resultados positivos. Diante disso, conclui-se que a nova equação do IMC pode ser considerada como um novo método para avaliar o estado nutricional de crianças e adolescentes de 10 a 14 anos de idade.

\section{REFERÊNCIAS}

1. Nahas MV. Atividade física, saúde e qualidade de vida. Londrina: Midiograf; 2003.

2. Carvalho EAA, Simão MTJ, Fonseca MC, Andrade RG, Ferreira MSG, Silva AF. et al. Obesidade: aspectos epidemiológicos e prevenção. Rev. Assoc. Méd. Minas Gerais. 2013;23(1):74-82.

3. Souza WC, Mascarenhas LPG, Tajes Junior D, Muniz MAB, Brasilino FF. Associação entre o IMC, \%G e a RCQ em meninas escolares. Revista Brasileira de Inovação Tecnológica em Saúde. 2014;4(4):54-61.

4. Queiroga MR. Testes e medida para avaliação da aptidão física relacionada à saúde em adultos. Rio de Janeiro: Guanabara Koogan; 2005.

5. Sant'Anna MSL, Priore SE, Franceschini SCC. Métodos de avaliação da composição corporal em crianças. Rev Paul Pediatr. 2009;27(3):31521.

6. Gonçalves R, Mascarenhas LPG, Liebl EC, Lima VA, Souza WB, Grzelczak MT. et al. Grau de concordância do IMC e do IAC com percentual de gordura. Revista Brasileira de Qualidade de Vida. 2014;6(1):8-16.

7. Buss V, Franz LBB, Steinmetz SB, Manenti M, Weber ITS, Conte FA. Estado nutricional: análise de uma nova proposta de equação. Resumo no Salão do Conhecimento; 2013; ljuí, Rio Grande do Sul, Brasil. 
8. Trefethen LN. (BMI) Body mass index [Internet]. Oxford: Mathematical Institute of University of Oxford; 2013 [cited 2016 Feb 12]. Available from: https://people.maths.ox.ac.uk/trefethen/ bmi.html

9. Petroski EL. Antropometria: técnicas e padronizações. [São Paulo]: Fontoura; 2011.

10. Quételet LAJ. Antropométrie ou mesure des différentes facultés de l'homme. Bruxelles: Muquardt; 1870.

11. Center for Disease Control and Prevention. Body Mass Index: Considerations for Practitioners. [cited 2016 Apr 04]. Available from: http://www.cdc.gov/obesity/downloads/ BMlforPactitioners.pdf

12. Lohman TG. Applicability of body composition techniques and constants for children and youths. Exerc Sports Sci Rev. 1986;14:325-57.

13. Gomes LPS, Leite SP, Lemos CFS, Lessa KMR, Pedral RL, Brandão PP. Métodos de obtenção de dados antropométricos confiáveis. Ciências Biológicas e de Saúde. 2015;3(1):87-100.

14. Lohman TG. The use of skinfold to estimate body fatness on children and youth. Journal of Physical Education, Recreation \& Dance. 1987;58(9):98-103.
15. Westphal P, Ferreira C, Adamczeski M, Camargo L, Santos R, Massaneiro AC. et al. Relação entre índice de massa corporal de Quételet e o de Trefethen. Revista CPAQV. 2016;8(3):1-6.

16. Beaudin L, Skaza J. Measuring the total impact of demographic and behavioural factors on the risk of obesity accounting for the depression status: a structural model approach using new BMI. Appl Econ. 2015;47(55):6041-53.

17. Blackburn H, Jacobs Jr D. Commentary: Origins and evolution of body mass index (BMI): continuing saga. Int J Epidemiol. 2014;43(3):665-9.

18. Santos AF, Maeda VA, Salomão PT. Desenvolvimento de melhorias para o sistema de avaliação e acompanhamento de atividades físicas. Anais do III Seminário de Iniciação Científica e Tecnológica; 2015 out 19-2O; Ponta Porã, Mato Grosso do Sul, Brasil.

19. Nunes LG. Análises dos índices antropométricos e hemodinâmicos relacionados às doenças cardiovasculares em crianças pré-púberes praticantes de atividade física. Revista NorteMineira de Educação Física. 2014;4(4):5-10.

2O. Lohman TG. Advances in Body Composition Assessment: Current Issues in Exercise Science. Champaign, Illinois: Human Kinetics Publishers; 1992. Monograph 3. 